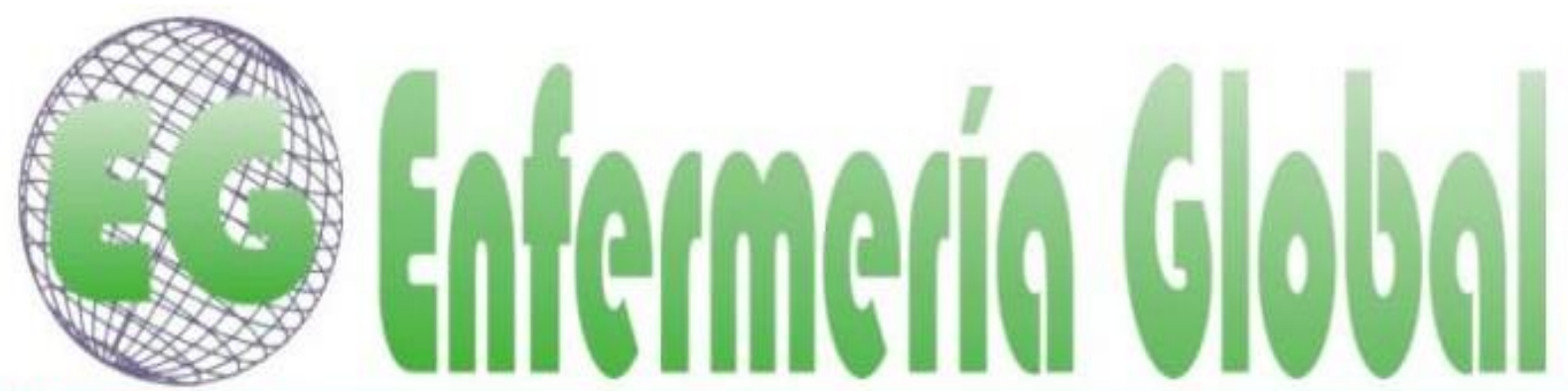

\title{
Efecto del temperamento infantil en el peso del niño preescolar: una revisión sistemática
}

Effect of infant temperament on the weight of preschool children: a systematic review

\section{*Amaro Hinojosa, Marily Daniela **Flores Peña, Yolanda}

\author{
*Estudiante de Doctorado de la Facultad de Enfermería de la Universidad Autónoma de Nuevo León. \\ E-mail: marily.amaroh@yahoo.com.mx **Profesor a tiempo completo de la Facultad de Enfermería \\ de la Universidad Autónoma de Nuevo León. México.
}

http://dx.doi.org/10.6018/eglobal.16.2.262231

\section{RESUMEN}

Antecedentes: El temperamento es una variable que recientemente ha resultado de interés por considerarse un factor de riesgo de la obesidad infantil. Algunos estudios señalan que el temperamento difícil se asocia al incremento del peso en los preescolares.

Objetivo: Analizar la literatura disponible sobre la asociación entre el temperamento infantil y el IMC del niño preescolar.

Métodos: Se realizó una revisión de la literatura sobre estudios que evaluaran la asociación entre el temperamento e índice de masa corporal en el niño preescolar, la búsqueda se realizó en los meses de mayo y junio del 2016, en bases de datos como Pubmed, EBSCO host, Ovid, Springer Link y Google académico, se incluyeron estudios que no tuvieran más de 10 años de haberse publicado y que cumplieran con los criterios de inclusión y calidad en la metodología y resultados presentados, como referencia para la evaluación de los estudios se utilizó la guía Critical Apprasisal Skills Programme Español.

Resultados: Se encontraron 784 artículos, de los cuales solo nueve se incluyeron para esta revisión, seis de los estudios evaluaban el temperamento en base a la reactividad atencional y/o rasgos, tres evaluaban el temperamento en base a la autorregulación del niño y solo uno evaluó tanto la reactividad emocional como la autorregulación. Pareciera que la reactividad negativa y la baja autorregulación son factores asociados a la obesidad infantil en la edad preescolar.

Conclusiones: La literatura encontrada sugiere que es necesaria mayor investigación para determinar el comportamiento del temperamento en torno a la obesidad en los niños.

Palabras clave: Temperamento; Obesidad pediátrica; Índicee de masa corporal 


\title{
ABSTRACT
}

Background: Temperament is a variable that has recently been shown to be of interest as a risk factor for childhood obesity. Some studies indicate that difficult temperament is associated with weight gain in preschoolers.

Objectives: The aim of the present study was to analyze the available literature on the association between the child's temperament and the BMI of the preschool child.

\begin{abstract}
Methods: We did a review of the literature about studies evaluating the association amoung temperament and body mass index in the preschool child. The search was carried since May to June of 2016 in databases such as Pubmed, EBSCO Host, Ovid, Springer Link and academic google. We included studies that had not more than 10 years of published and that complied criteria for inclusion, in addition the results of the studies had quality in the methodology and results, as reference for the evaluation of the studies, we used The Critical Apprasisal Skills Program English.
\end{abstract}

Results: We found 784 articles, of which only nine were included for this review, six of the studies evaluated temperament based on attentional reactivity and / or traits, three evaluated temperament based on self-regulation of the child and only one Evaluated both emotional reactivity and selfregulation. It seems that negative reactivity and low self-regulation are factors associated with childhood obesity in the preschool age.

Conclusions: The literature suggests that more research is needed to determine the temperament behavior around obesity in children.

Keywords: Temperament; Pediatric obesity, Body Mass Index

\section{INTRODUCCIÓN}

La obesidad (OB) infantil se considera un problema alarmante en la salud, ya que de no ser tratada a tiempo, puede generar enfermedades como la diabetes mellitus, enfermedades cardiovasculares, cáncer, enfermedades del aparato locomotor, ansiedad, depresión e incluso moralidad prematura, lo que además desencadena un impacto en la economía de los países, por el gasto anual en el tratamiento de las complicaciones de las enfermedades generadas por la OB y por la pérdida de fuerza laboral en edades cada vez más tempranas ${ }^{(1)}$. Diversa investigación continúa en la búsqueda del entendimiento del incremento del peso en la infancia, ya que a pesar de saber que los principales factores de riesgo para el Sobrepeso (SP) y OB son el consumo de alimentos con alto contenido calórico y la insuficiente actividad física, la literatura reconoce que existen múltiples factores genéticos y ambientales que intervienen para que un niño presente o no SP y OB ${ }^{(2)}$.

Durante los primeros 5 años de vida, es cuando se aprenden las conductas de alimentación, preferencias y rechazos a determinados alimentos y la actividad física suficiente ${ }^{(3)}$, las cuales son aprendidas por los hijos a través de la madre, principal modelo y promotor de conductas en el hijo. Sin embargo, también existen características biológicas, genéticas y psicológicas del niño que pueden influir en las decisiones de los padres sobre las conductas de alimentación que promueven, de no ser saludables pueden trascender hasta el SP-OB infantil. Un ejemplo es el temperamento infantil, el cual se ha asociado al rápido incremento de peso en los niños lactantes y preescolares, pues las madres que reportan tener niños con temperamento difícil, refieren que los alimentan frecuentemente para lograr tranquilizarlos, es decir, la característica del hijo favorece a la promoción de conductas no saludables, como la sobrealimentación por parte de la madre ${ }^{(4,5)}$.

El temperamento puede definirse como un rasgo estable y duradero, que tiene bases biológicas, además está vinculado a la dotación genética de cada individuo y 
determina las respuestas afectivas, de atención y motoras en diferentes situaciones, las manifestaciones de la conducta del temperamento, son modificadas a través del tiempo, debido a la interacción con los padres ${ }^{(6,7)}$. El temperamento constituye diferencias entre dos componentes: la reactividad atencional y la autorregulación.

1) La reactividad se refiere a las características de las reacciones individuales dadas por lo cambios en el ambiente que se reflejan a través de respuestas del sistema somático, emocional, endocrino y nervioso; misma que puede ser: a) negativa, cuando el niño expresa y siente angustia y presenta un comportamiento y aversión atencional $\circ$ b) positiva, el niño expresa y siente afecto positivo y comportamiento y enfoque atencional.

2) La autorregulación incluye procesos de funcionamiento que modulan la reactividad de la persona como por ejemplo: los patrones atencionales: el enfoque y la evitación (8). El desarrollo de la autorregulación resulta del incremento de control sobre el proceso atencional, así como del control inhibitorio sobre la conducta motora. Dos habilidades de autorregulación se han identificado: la regulación emocional y la demora en la recompensa ${ }^{(9)}$.

En este sentido, la literatura ha documentado cómo los diferentes tipos de temperamento y sus procesos pueden afectar a que el niño esté consumiendo alimentos que sobrepasan sus necesidades energéticas, lo que tendrá como resultado un incremento en su peso. El temperamento se ha evaluado de diferentes formas, a través de rasgos, los cuales reflejan las respuestas que se dan de la reactividad atencional. En este sentido, un estudio encontró que el rasgo de habilidad para autocalmarse y la no capacidad de atención fueron predictores del exceso de peso en las niñas y niños preescolares respectivamente ${ }^{(10)}$. Otro estudio, demostró que el temperamento difícil (reactividad negativa) del hijo está asociado con su exceso de peso, problemática que se engrandece cuando la madre alimenta a su hijo frecuentemente como medida para calmarlo ${ }^{(5)}$. Otros estudios evalúan la autorregulación, un estudio encontró que niños con bajo control inhibitorio (baja autorregulación) ${ }^{(11)}$ y baja demora en la recompensa ${ }^{(9-11)}$ tienen menor habilidad de utilizar estrategias para redirigir sus preferencias a los alimentos deseables, lo que se traduce a un incremento de peso a través del tiempo. Sin embargo, existen estudios que refieren que el temperamento infantil no se asocia al incremento de peso en la edad preescolar ${ }^{(12-13)}$.

La literatura existente reporta que el temperamento es una variable de interés que pudiera ayudar a una mayor comprensión del fenómeno de la obesidad en los preescolares, por lo que se considera de importancia realizar una revisión en la que se conjunten aquellos estudios que brinden información teórica y metodológica sobre el temperamento en la edad preescolar y el efecto que tiene en el índice de masa corporal (IMC) del niño, ya que bajo nuestro conocimiento solo se encontró una revisión sistemática que además incluía la variable prácticas de alimentación ${ }^{(12)}$. Sin embargo, nuestro interés es ver si existe una asociación directa entre el temperamento y el IMC del niño preescolar, independientemente de otras variables moderadoras, por lo que el presente estudio tiene el objetivo de analizar y sintetizar la literatura existente sobre la variable temperamento infantil (es decir, evalúe reactividad atencional, la autorregulación o ambas) y su asociación con el SP-OB del niño en etapa preescolar en donde la variable resultado sea el IMC del niño. 


\section{METODOLOGÍA}

Esta revisión sistemática usó como guía base la lista de chequeo propuesta por el grupo PRISMA de 27 reactivos ${ }^{(14)}$. Por lo que este trabajo se identificó como una revisión sistemática, previamente se realizó una revisión de la literatura para poder formular la importancia y el objetivo de esta revisión, mismo que siguió los parámetros propuestos por PICOS (Paciente, intervención, comparación, resultados y diseño de estudio).

Se establecieron los criterios de inclusión: estudios que evaluaran la correlación del temperamento y la obesidad infantil, en donde la variable resultado fuera IMC del niño, niños en edades entre 3 a los 6 años. Los diseños de estudios que se incluyeron fueron descriptivos correlacionales, longitudinales, revisiones sistemáticas 0 metanálisis y estudios disponibles en idioma inglés y español. Se consideró literatura publicada de no más de 10 años. Posteriormente se seleccionaron las bases de datos electrónicas para realizar la búsqueda: PubMed, EBSCO host (Medline, Health Source, Nursing/Academic edition, Medic Latina, CINAHL), Ovid, Springer link, además del buscador Google académico.

Respecto a la búsqueda, se identificaron las palabras clave de los Descriptores de Ciencias de la Salud DeCs y Mesh: pediatric Obesity, childhood obesity, child obesity, temperament, body mass index. Además de las palabras clave, se utilizaron los operadores boléanos "AND", "OR" y "NOT" y algunos truncadores.

[Cuadro 1 Estrategia de búsqueda]

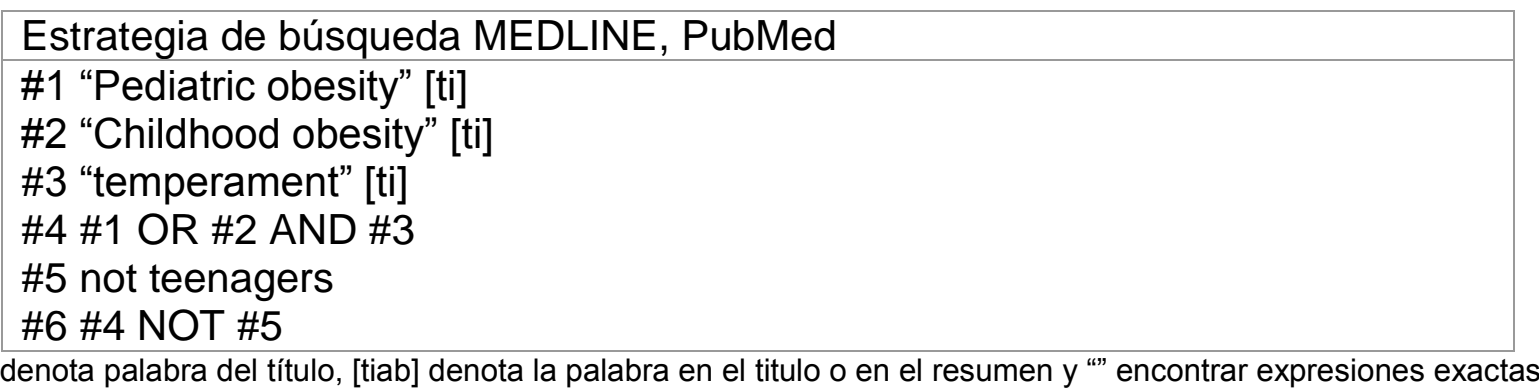

Se siguió el siguiente procedimiento para la selección de los estudios: primero se colocaron las palabras clave y los operadores boléanos en las bases de datos seleccionados, de los estudios arrojados, se eliminaron las citas duplicadas, para luego seleccionar aquellos que incluían en el titulo las variables de nuestro interés, posteriormente se evaluó el resumen de los artículos restantes, aquellos artículos que no cumplían con los criterios de inclusión fueron excluidos, se evaluó a texto completo los estudios restantes a través de dos métodos: por apartados y por preguntas de la plantilla CASPe (Critical Apprasisal Skills Programme Español). Por apartados se revisó lo siguiente: el título debía ser claro y correctamente redactado e incluir las variables a investigar; el resumen como mínimo tenía que mostrar el objetivo, métodos, resultados y conclusiones, los cuales debían de ser congruentes y el artículo debía incluir palabras clave.

La introducción debía presentar un adecuado planteamiento del problema, estar contextualizado y finalizaría con un objetivo, hipótesis o propósito; con respecto a la metodología tendría que tener un diseño que respondiera al objetivo del estudio, la muestra significativa, aleatoriedad, descripción de los instrumentos utilizados y el 
análisis de datos fuera congruente con el diseño y objetivo del estudio. Los resultados mostraran respuesta a los objetivos y las conclusiones mostraran las limitaciones del estudio. Respecto a la plantilla CASPe se realizaron las preguntas: $¿ E$ El resultado se midió de forma precisa con el fin de minimizar posibles sesgos?, ¿Han tenido en cuenta los autores el potencial efecto de los factores de confusión en el diseño y/o análisis del estudio?, ¿Los resultados de este estudio coinciden con otra evidencia disponible?

\section{RESULTADOS}

Se encontraron un total de 784 estudios, de los cuales se excluyeron 7 por ser citas duplicadas, de los 777 estudios restantes, solo se seleccionaron 34 estudios por contener en el titulo las variables de interés para esta revisión, se eliminaron 21 por no cumplir con los criterios de inclusión establecidos, por lo que se evaluó a texto completo 13 de los artículos, posteriormente se eliminaron 4 por no cumplir con los criterios de calidad en la metodología y/o análisis de los resultados presentados y finalmente para esta revisión sistematica se incluyeron 9 estudios, se anexa el diagrama prisma (figura 1.1).

[Figura 1 Diagrama Prisma, utilizado para la selección de los artículos incluidos en la revisión sistemática]

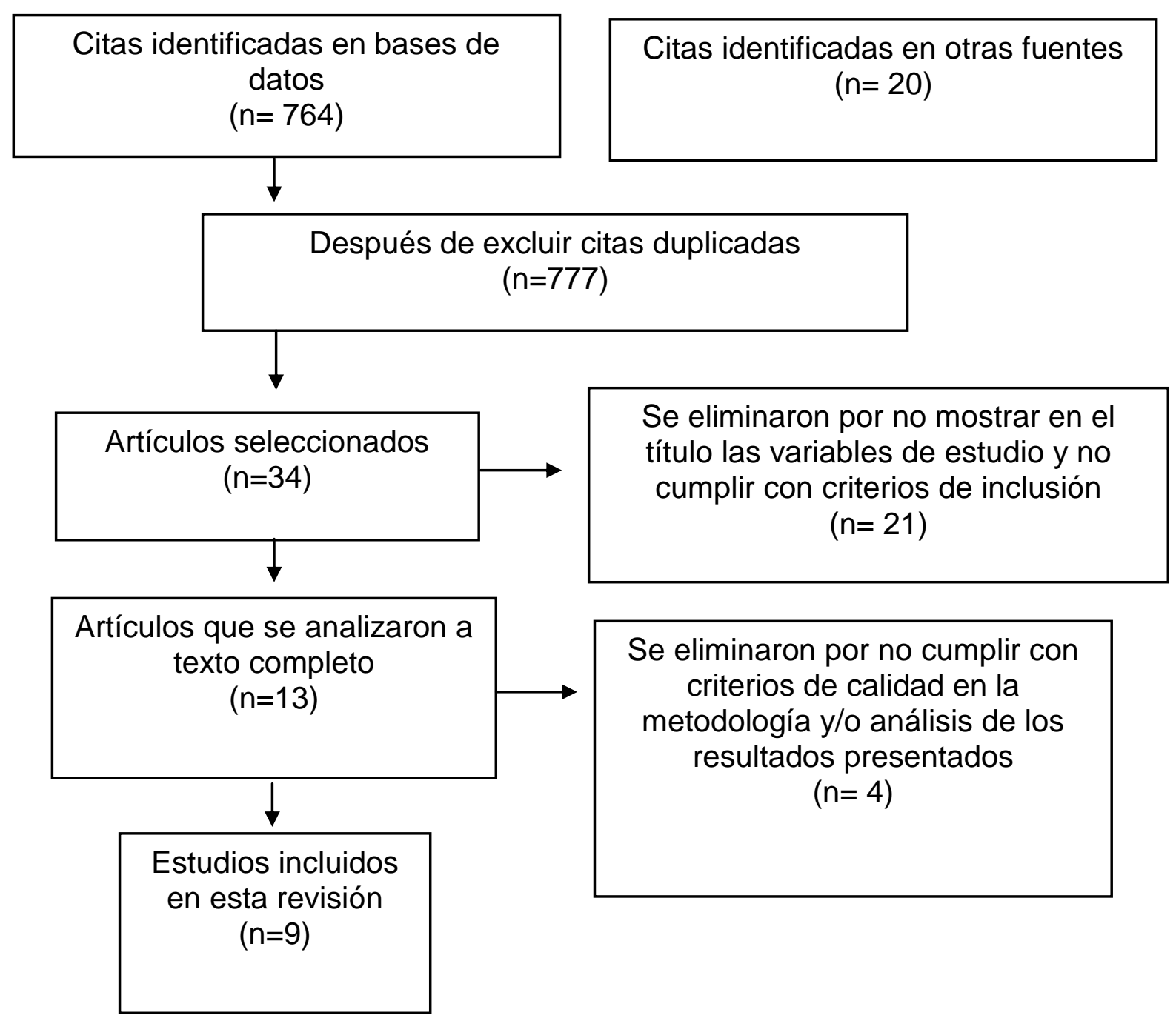




\section{Características de los estudios}

De los nueve estudios revisados, siete fueron realizados en Estados Unidos $^{(5,9,10,11,15,16,17)}$, uno en Australia ${ }^{(12)}$ y uno en el Reino Unido ${ }^{(13)}$. Respecto al diseño, tres de los estudios fueron de tipo transversal $(13,15,16)$ y seis eran longitudinales ${ }^{(5,9,10,11,12,17)}$, estos estudios tenían de seguimiento de 2 hasta 10 años.

En cuanto a la muestra, los participantes eran de origen caucásico, afroamericano e hispano, la mayoría de los estudios midieron a las diadas madres e hijo ${ }^{(12,10,5,13,9)}$, otros realizaban las mediciones en ambos padres y en el hijo ${ }^{(15,16,17)}$ y uno solo estudio incluyo solo a los niños ${ }^{(11)}$. Los estudios utilizaron para la clasificación del IMC del niño, la edad y los percentiles según los parámetros establecidos por el centro de control de enfermedades por sus siglas en inglés $\operatorname{CDC}^{(5,9,10,11,12,15,16,17)}$ y solo un estudio realizó la clasificación del IMC según la Child Growth Foundation reference curves disc ${ }^{(13)}$.

Para la presentación de los hallazgos individuales de los estudios, se realizó una tabla de resultados.

[Tabla I Análisis individual de los datos de los estudios seleccionados] 
[Tabla I Análisis individual de los datos de los estudios seleccionados]

\begin{tabular}{|c|c|c|c|c|}
\hline Autor- año & $\begin{array}{l}\text { Diseño de } \\
\text { estudio }\end{array}$ & Muestra & Intumento de medición & $\begin{array}{l}\text { Asociación entre el } \\
\text { temperamento y el IMC del } \\
\text { niño }\end{array}$ \\
\hline $\begin{array}{l}\text { Bergmeier, } \\
2014^{(12)}\end{array}$ & longitudinal & $\begin{array}{l}201 \\
\text { Diadas } \\
\text { de } \\
\text { madres e } \\
\text { hijos (2-5 } \\
\text { años) }\end{array}$ & $\begin{array}{l}\text { The short temperament } \\
\text { scale } \\
\text {-Cooperación } \\
\text {-Irritabilidad } \\
\text {-Enfoque } \\
\text { Confiabilidad: }(\alpha=0.82-0.85)\end{array}$ & $\begin{array}{l}\text { No se encontró asociación } \\
\text { significativa }\end{array}$ \\
\hline $\begin{array}{l}\text { Graziano, } \\
2013^{(9)}\end{array}$ & longitudinal & $\begin{array}{l}195 \\
\text { niños y } \\
\text { sus } \\
\text { madres } \\
(2,4,5,7 \text { y } \\
10 \text { años) }\end{array}$ & $\begin{array}{l}\text { Toddler behavior } \\
\text { assessment questionnaire } \\
\text { Dimensiones: } \\
\text {-Nivel de actividad } \\
\text {-Placer } \\
\text {-Temor social } \\
\text {-Propenso a ira } \\
\text {-Interés/persistencia } \\
\text { Confiabilidad ( } \alpha=0.84 \text { ) } \\
\text { Regulación emocional: } \\
\text {-Se colocó en una caja } \\
\text { cerrada un juguete preferido } \\
\text { del niño y se le pidió que } \\
\text { esperara por } 2 \text { minutos } \\
\text { Se colocó al niño en una } \\
\text { silla alta, sin ningún juguete } \\
\text { por } 5 \text { minutos } \\
\text { (Kappas .80) (r= -.91, } \\
\text { p<.001) } \\
\text { Sensibilidad de recompensa } \\
\text {-Se midió el tiempo que } \\
\text { tardo el niño en acercarse y } \\
\text { abrir una caja envuelta de } \\
\text { forma atractiva, la cual se le } \\
\text { dijo que era un regalo para } \\
\text { él } \\
\text { ( } r=.99 \text { ) } \\
\text { Atención sostenida } \\
\text {-Se colocó un video de } 5 \\
\text { minutos y se midió el tiempo } \\
\text { que el niño paso viendo un } \\
\text { video, sin perder la atención } \\
\text { ( } r=.98 \text { ) }\end{array}$ & $\begin{array}{l}\text { A los } 4 \text { años se reportó una } \\
\text { relación significativa entre el } \\
\text { temperamento/ habilidades } \\
\text { de autorregulación } \\
\text { (regulación emocional, } \\
\text { sensibilidad de la } \\
\text { recompensa y atención } \\
\text { sostenida) las cuales fueron } \\
\text { predictores positivos del IMC } \\
\text { a los } 10 \text { años ( } \beta=0.31, p \\
<0.0001 \text { ) }\end{array}$ \\
\hline $\begin{array}{l}\text { Faith, } 2010 \\
(10)\end{array}$ & longitudinal & $\begin{array}{l}487 \\
\text { diadas } \\
\text { madre e } \\
\text { hijo } \\
\text { (1 hasta } \\
6 \text { años) }\end{array}$ & $\begin{array}{l}\text { Colorado child } \\
\text { Temperament Inventory } \\
\text { (Rowe \& Plomin,1977) } \\
\text {-Emocionalidad } \\
\text {-Actividad } \\
\text {-Capacidad de atencion } \\
\text {-Reaccion a la comida } \\
\text {-Habilidad para } \\
\text { autocalmarse (10 ítems) } \\
\text {-Reactividad de los } \\
\text { alimentos (5 ítems) } \\
\text {-Apetito predecible ( } 3 \text { ítems) } \\
\text {-Distracción a la hora de } \\
\text { comer (2 ítems) } \\
\text { Confiabilidad ( } \alpha=0.78)\end{array}$ & $\begin{array}{l}\text { En los niños la capacidad de } \\
\text { atención fue significativa } \\
\text { como predictor de la } \\
\text { ganancia de peso } \\
\text { En las niñas la reactividad a } \\
\text { los alimentos y habilidad de } \\
\text { autocalmarse fueron } \\
\text { significativas para la } \\
\text { ganancia de peso }\end{array}$ \\
\hline
\end{tabular}




\begin{tabular}{|c|c|c|c|c|}
\hline Autor- año & $\begin{array}{l}\text { Diseño de } \\
\text { estudio }\end{array}$ & Muestra & Intumento de medición & $\begin{array}{l}\text { Asociación entre el } \\
\text { temperamento y el IMC del } \\
\text { niño }\end{array}$ \\
\hline $\mathrm{Wu}, 2011^{\text {(5) }}$ & longitudinal & $\begin{array}{l}1201 \\
\text { madres e } \\
\text { hijos (2- } \\
12 \text { años) }\end{array}$ & $\begin{array}{l}\text { Infant temperament } \\
\text { questionnaire } \\
\text {-Enfoque } \\
\text {-Actividad } \\
\text {-Intensidad } \\
\text {-Estado de animo } \\
\text {-Adaptabilidad } \\
\text { Confiabilidad ( } \alpha=0.81 \text { ) } \\
\\
\text { Los niños fueron agrupados } \\
\text { en las tres categorías (niño } \\
\text { difícil, fácil y tímido) } \\
\text { 1. Niño fácil: se define como } \\
\text { alta Ritmicidad, enfoque y } \\
\text { adaptabilidad, bajo en } \\
\text { intensidad y estado de } \\
\text { ánimo positivo } \\
\text { 2. Niño difícil: baja } \\
\text { ritmicidad, enfoque y } \\
\text { adaptabilidad, alta } \\
\text { intensidad y estado de } \\
\text { ánimo negativo } \\
\text { 3. Niños lentos para } \\
\text { reaccionar/niños tímidos: } \\
\text { baja actividad, enfoque } \\
\text { adaptabilidad, alta } \\
\text { intensidad y estado de } \\
\text { ánimo negativo }\end{array}$ & $\begin{array}{l}\text { No se reportó relación entre } \\
\text { el tipo de temperamento del } \\
\text { niño y el IMC, fue hasta que } \\
\text { se agregó la variable } \\
\text { sensibilidad/insensibilidad } \\
\text { de la madre, cuando se } \\
\text { mostraron estadísticos } \\
\text { significativos } \\
\text { Se encontró una relación } \\
\text { entre un alto IMC del niño } \\
\text { en edad escolar ( } 5 \text { a } 12 \\
\text { años), ser un niño tímido y } \\
\text { tener una madre insensible } \\
\text { ( } \beta=4.756, p<0.01) \\
\text { Se reportó una relación } \\
\text { entre un alto IMC del niño } \\
\text { en edad escolar (5 a } 12 \\
\text { años), ser un niño difícil y } \\
\text { tener una madre insensible } \\
\text { ( } \beta=4.866, p<0.05)\end{array}$ \\
\hline $\begin{array}{l}\text { Hughes, } \\
2008^{(15)}\end{array}$ & transversal & $\begin{array}{l}718 \\
\text { padres } \\
\text { de niños } \\
\text { (3- } 5 \\
\text { años) }\end{array}$ & $\begin{array}{l}\text { The children's behavior } \\
\text { Questionnaire (very short } \\
\text { form) (CBQ) } \\
\text {-Afectividad negativa } \\
\text { ( } \alpha=0.65) \\
\text {-Control de esfuerzo } \\
\text { ( } \alpha=0.74) \\
\text {-Extroversión/Insurgencia } \\
\text { ( } \alpha=0.63)\end{array}$ & $\begin{array}{l}\text { Se realizó un análisis } \\
\text { multivariado de la varianza } \\
\text { entre las variables: } \\
\text { asociadas con el peso del } \\
\text { niño (etnia, género del hijo e } \\
\text { IMC parental), demográficas } \\
\text { (educación parental, edad } \\
\text { parental y edad del hijo), } \\
\text { psicosociales } \\
\text { (temperamento del hijo y } \\
\text { afecto de la madre) y el } \\
\text { estilo indulgente de } \\
\text { alimentación, el modelo } \\
\text { predijo el IMC del hijo en un } \\
12 \% \text {. }\end{array}$ \\
\hline $\begin{array}{l}\text { Haycraft, } \\
2011^{(13)}\end{array}$ & transversal & $\begin{array}{l}241 \\
\text { diadas } \\
\text { (madres } \\
\text { e hijo de } \\
\text { edades } \\
\text { de 3-8 } \\
\text { años) }\end{array}$ & $\begin{array}{l}\text { Temperament Survey for } \\
\text { children (EAS) } \\
\text {-Tímido } \\
\text {-Emocionalidad } \\
\text {-Sociabilidad } \\
\text {-Actividad } \\
\text { Confiabilidad ( } \alpha=0.58-0.83 \text { ) }\end{array}$ & $\begin{array}{l}\text { El IMC del niño no fue } \\
\text { significativo con ninguno de } \\
\text { los rasgos de temperamento }\end{array}$ \\
\hline $\begin{array}{l}\text { Graziano, } \\
2010^{(11)}\end{array}$ & longitudina & $\begin{array}{l}57 \text { niños } \\
\text { (de } 2 \text { a } \\
5.5 \text { años) }\end{array}$ & $\begin{array}{l}\text { Regulación emocional: } \\
\text {-Se colocó en una caja } \\
\text { cerrada un juguete preferido } \\
\text { del niño y se le pidió que } \\
\text { esperara por } 2 \text { minutos } \\
\text { Se colocó al niño en una } \\
\text { silla alta, sin ningún juguete } \\
\text { por } 5 \text { minutos } \\
\text { (Kappas .80) } \\
\text { ( } r=-.91, p<.001) \\
\text { Atención sostenida } \\
\text {-Se colocó un video de } 5 \\
\text { minutos y se midió el tiempo } \\
\text { que el niño paso viendo un } \\
\text { video, sin perder la atención } \\
(r=.98)\end{array}$ & $\begin{array}{l}\text { La regulación emocional fue } \\
\text { un predictor significativo del } \\
\text { IMC del niño a los } 5.5 \text { años } \\
(\beta=-.41, p<.01) \\
\text { El control } \\
\text { inhibitorio/sensibilidad de } \\
\text { recompensa también fue un } \\
\text { predictor significativo del } \\
\text { peso del niño a los } 5.5 \text { años } \\
(\beta=-.17, p<.05)\end{array}$ \\
\hline
\end{tabular}




\begin{tabular}{|c|c|c|c|c|}
\hline Autor- año & $\begin{array}{l}\text { Diseño de } \\
\text { estudio }\end{array}$ & Muestra & Intumento de medición & $\begin{array}{l}\text { Asociación entre el } \\
\text { temperamento y el IMC del } \\
\text { niño }\end{array}$ \\
\hline & & & $\begin{array}{l}\text { Sensibilidad de recompensa } \\
\text {-Se midió el tiempo que } \\
\text { tardo el niño en acercarse y } \\
\text { abrir una caja envuelta de } \\
\text { forma atractiva, la cual se le } \\
\text { dijo que era un regalo para } \\
\text { él }(r=.99)\end{array}$ & \\
\hline $\begin{array}{l}\text { Tan, } \\
2010^{(16)}\end{array}$ & transversal & $\begin{array}{l}63 \\
\text { padres e } \\
\text { hijos (3-9 } \\
\text { años) }\end{array}$ & $\begin{array}{l}\text { Autorregulación alimentaria } \\
\text {-Se incluyeron } 8 \text { ítems } \\
\text { desarrollados en base a la } \\
\text { revisión de literatura } \\
\text { existente } \\
\text { Por ejemplo: Mi hijo sabe } \\
\text { cuanta comida debería } \\
\text { comer, mi hijo deja de comer } \\
\text { cuando se siente lleno, etc. } \\
\text { Confiabilidad ( } \alpha=0.87 \text { ) } \\
\text { Control inhibitorio } \\
\text { The children's behavior } \\
\text { questionnaire (CBQ) } \\
\text { Subescala control de } \\
\text { esfuerzo } \\
\text { Confiabilidad ( } \alpha=0.85 \text { ) }\end{array}$ & $\begin{array}{l}\text { Se encontró una relación } \\
\text { negativa y significativa entre } \\
\text { la autorregulación } \\
\text { alimentaria y el IMC del hijo } \\
(\mathrm{r}=-.29, \mathrm{p}=.05) .\end{array}$ \\
\hline $\begin{array}{l}\text { Francis, } \\
2009^{(17)}\end{array}$ & longitudinal & $\begin{array}{l}1061 \\
\text { familias y } \\
\text { sus hijos } \\
(3,5,7,9,1 \\
1 \text { y } 12 \\
\text { años) }\end{array}$ & $\begin{array}{l}\text {-Procedimiento para medir el } \\
\text { autocontrol en niños de } 3 \\
\text { años } \\
\text {-La estimación de entre } \\
\text { calificadores fiabilidad fue de } \\
0.92 \text {. } \\
\text {-Procedimiento para medir } \\
\text { retraso en la gratificación }\end{array}$ & 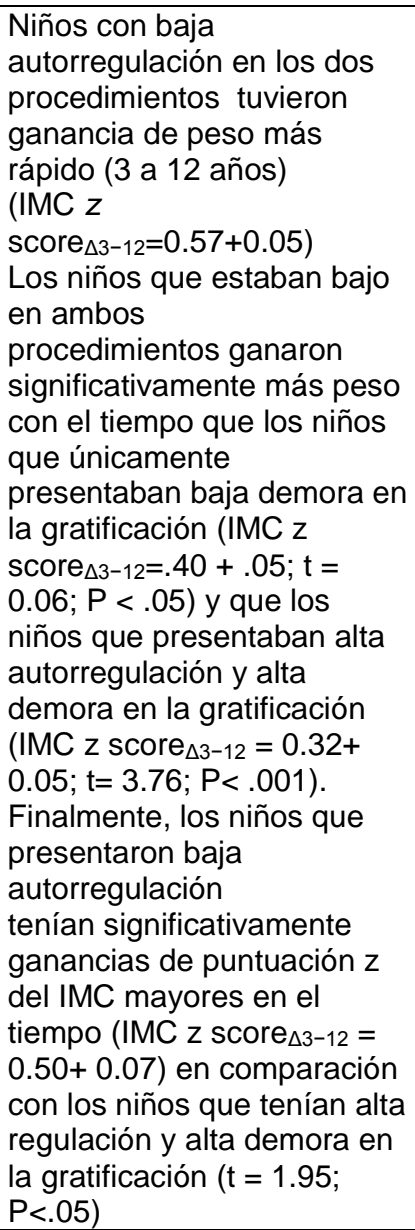 \\
\hline
\end{tabular}

Para la síntesis de los resultados de los estudios seleccionados se tomó la decisión de dividirlos en dos categorías, aquellos que reportaban el temperamento a través de la reactividad/rasgos y autorregulación. 


\section{Temperamento (Reactividad/rasgos)}

Seis de los estudios evaluaron la reactividad atencional y los rasgos del temperamento. Respecto al sustento teórico de los estudios, tres de ellos utilizaron la definición dada por Rothbard y sus colaboradores, en la cual establecen que el temperamento es un rasgo estable y duradero, que tiene bases biológicas, además está vinculado a la dotación genética de cada individuo y determina las respuestas afectivas, de atención y motoras en diferentes situaciones (citado por 9, 10,5). Un estudio utilizó la definición propuesta por Sanson (1987) (citado por 12), quien establece tres dimensiones para evaluar el temperamento: la emotividad/afectividad, autorregulación/control de esfuerzo y afecto/ enfoque positivo ${ }^{(12)}$. Un estudio hace referencia a la definición dada por Buss y Plomin's (1977), para ellos el temperamento infantil puede clasificarse en cuatro rasgos: timidez, emocionalidad, sociabilidad y

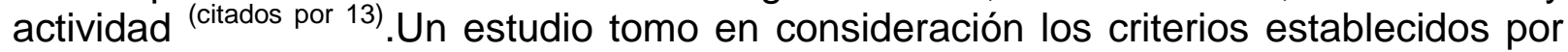
Sadness quien sugiriere que las dimensiones del temperamento son tres: afectividad negativa, control de esfuerzo y extroversión/insurgencia (citado por 15). Cabe señalar que un estudio reportó a más de un autor como sustento teórico del temperamento, e hizo uso de la definición propuesta por Rothbard, las dimensiones del temperamento propuestas por Thomas y Chess (nivel de actividad, ritmicidad, enfoque/retracción, adaptabilidad, intensidad, estado de ánimo, persistencia/ capacidad de atención, distracción y el umbral de la capacidad de respuesta) y las tipologías utilizadas por Dixon y Smith (2000) Maziade, Caron, Cote y Merette (1990) para finalmente clasificar a los niños como: niño fácil, niño difícil y niño tímido (citados por 5).

Respecto a los instrumentos de medición utilizados se encuentran: 1) The short temperamente scale $\left.{ }^{(12)}, 2\right)$ Toddler behavior assessment questionnaire $\left.{ }^{(9)}, 3\right)$ Colorado child temperament inventory ${ }^{(10)}$, 4) Infant temperament questionnaire ${ }^{(5)}$, 5) The children's behavior questionnaire $(\mathrm{CBQ}){ }^{(15)}$ y 6 ) Temperament survey for childrens ${ }^{(13)}$, todos ellos con confiabilidad aceptable.

En cuanto a la relación entre el temperamento y el SP-OB infantil. Dos de los estudios no encontraron una asociación significativa entre los rasgos del temperamento y el IMC del niño ${ }^{(12,13)}$. Un estudio encontró que niños que presentaban el rasgo de la expresión intensa al placer se asoció con el de peso del niño a través del tiempo ${ }^{(9)}$. Otro estudio refiere que la no capacidad de atención en los niños fue un predictor para el incremento de peso, pero no en las niñas, pues en ellas la reactividad a los alimentos (aquellas que les disgustaban muchos tipos de comida) y la baja habilidad para autocalmarse fueron los rasgos que se asociaron significativamente con el aumento de peso ${ }^{(10)}$.

Otro de los estudios encontró asociación entre el tipo de temperamento del niño y el aumento en el IMC, pero solo cuando estaba presente la variable sensibilidad de la madre, se encontró que existía un alto IMC cuando el niño era tímido o difícil y tenía una madre insensible ${ }^{(5)}$. Finalmente uno de los estudios demostró que el temperamento del hijo en conjunto con otras variables (etnia, género del hijo e IMC parental, educación parental, edad parental y edad del hijo, afecto de la madre y el estilo indulgente de alimentación) predijeron el IMC del hijo en un $12 \%{ }^{(15)}$.

\section{Temperamento (Autorregulación)}

Cuatro de los estudios midieron el componente de autorregulación, respecto al sustento teórico que estos estudios utilizaron fue el concepto de Rothbard, quien 
define a la autorregulación como un proceso que se basa en la atención, el enfoque y la evitación e inhibición, que en conjunto buscan inhibir la reactividad. Además, refiere que existen habilidades de autorregulación: la regulación emocional y la demora de gratificación. La regulación emocional, puede tener como resultado la reactividad o regulación emocional, la reactividad emocional se refiere a la angustia, queja, agitación, llanto y rabietas. La regulación emocional es el uso eficaz de diversas estrategias, por ejemplo, la distracción (citado por 9, 11, 16,17).

El control atencional para la demora de gratificación se asocia a dos funciones cognitivas: atención sostenida y control inhibitorio. La atención sostenida, se refiere a la capacidad del niño de mantener su atención en estímulos específicos y el control inhibitorio es la habilidad de restringir las respuestas prepotentes que pueden ser inapropiadas en determinada situación (citado por 9, 11, 16,17). Dos estudios midieron el concepto de autorregulación alimentaria, la cual se basa en el uso de señales internas para decidir la cantidad a consumir en base a las necesidades energéticas, las cuales son diferentes en cada individuo ${ }^{(10,16)}$.

Las mediciones de la autorregulación utilizadas fueron en base a observaciones realizadas en espacios controlados/laboratorios, por ejemplo, dos de los estudios midieron la regulación emocional a través de dos procedimientos: 1) se colocó en una caja cerrada un juguete preferido del niño y se le pidió que esperara por lo menos dos minutos y 2) se colocó al niño en una silla alta, sin ningún juguete por cinco minutos $^{(2,7)}$, a través de estos procedimientos, el observador medía el tiempo y las reacciones del niño ante estas situaciones. Respecto al control atencional y la demora de gratificación, fue medido a través de dos procedimientos: 1) sensibilidad de recompensa: se midió del tiempo que tardó el niño en acercarse y abrir una caja envuelta de forma atractiva, la cual se le dijo que era un regalo para él, 2) la atención sostenida: se colocó un video de cinco minutos y se midió el tiempo que el niño pasó viendo este video, sin perder la atención ${ }^{(9,11)}$.

Otro estudio utilizo distintos procedimientos, para la medición de la autorregulación en el niño, el entrevistador les mostró a los niños un "juguete objetivo" que era muy atractivo y permitió que los niños lo tocaran y jugaran con él, antes de que comenzara el "juego de espera". Una vez que los niños tuvieron la oportunidad de jugar con el juguete el entrevistador les dijo a los niños que él o ella se iban a salir para hacer un trabajo en otra habitación. Pero les dijeron a los niños que no se les permitía tocar el juguete objetivo hasta que el entrevistador volviera, mientras tanto podrían jugar con otro de los juguetes que estaban disponibles en la habitación; los niños se quedaban solos con el juguete blanco por 150 segundos ${ }^{(17)}$.

En ese mismo estudio, se midió el retraso en la gratificación a través del siguiente procedimiento, se les mostro a los niños 3 alimentos: dulces $M$ \& $M$, galletas de animalitos y pretzels. Una vez que los niños identificaron su alimento preferido, pequeños y grandes montones de la comida eran colocados en una mesa delante de ellos. Se les dijo a los niños que podrían tener el pequeño montón de alimentos en cualquier momento que quisieran, siempre y cuando fuera después de que el entrevistador saliera de la habitación y ellos hicieran sonar una campana para el entrevistador volviera antes y así los niños pudieran comer de la pequeña pila. Si el niño quería quedarse con el montón grande de comida, ellos tenían que esperar hasta que el entrevistador regresara por el mismo, sin que ellos tocaran la campana ${ }^{(17)}$. 
Dos estudios midieron el control inhibitorio a través de la subescala control de esfuerzo del cuestionario de la conducta del niño por sus siglas en inglés CBQ, la cual mostró una confiabilidad aceptable. En cuanto a la relación entre del componente del temperamento autorregulación y SP-OB infantil, se reportó que la reactividad emocional, menor tiempo de la sensibilidad a la recompensa y menor tiempo en la atención sostenida fueron predictores de un mayor IMC en el niño a través del tiempo ${ }^{(9,11)}$. Otro de los estudios encontró una asociación entre baja autorregulación alimentaria y el alto IMC del niño ${ }^{(16)}$. Un estudio reportó que los niños con baja autorregulación y baja demora en la gratificación presentaban mayor ganancia en el IMC a través del tiempo que los que mostraban alta autorregulación y alta demora en la gratificación ${ }^{(17)}$.

\section{DISCUSIÓN}

Los estudios encontrados suponen que el temperamento infantil es una variable que pareciera tener una asociación con la obesidad infantil, ya que de los nueve estudios revisados siete establecen que estas variables están relacionadas, sin embargo dos de estos estudios indican que el temperamento y la presencia de características parentales y socioeconómicas predicen con mayor exactitud el IMC del preescolar. Situación que podría ser porque el temperamento del niño y su ambiente afectan las conductas alimentarias y en consecuencia su peso.

En este sentido, es importante señalar que la alimentación es un proceso biopsicosocial en el que se involucran mecanismos homeostáticos, sistemas de recompensa neural, motor, sensorial y la capacidad socioemocional ${ }^{(18)}$. Al ser el temperamento un modulador de los procesos de actividad, emocionalidad y sociabilidad podría generar que un niño con temperamento difícil lograra tener mayor predisposición a conductas de alimentación no saludables, esta situación puede ser potencializada si además los padres principales cuidadores de los prescolares les proporcionan un ambiente cultural, social y económico no promotor de conductas saludables (sedentarismo, acceso limitado a alimentos saludables), estilos indulgentes $^{(}$en la alimentación ${ }^{(15)}$, entre otros, lo que finalmente resulta en el incremento de peso en la niñez temprana. Por lo tanto al ser la OB un problema multicausal y complejo es necesario considerar los factores individuales, sociales y ambientales con la finalidad de dar una mayor explicación a este problema de salud ${ }^{(1)}$.

En el caso de aquellos que encontraron resultados contradictorios, sería importante considerar los instrumentos de medición utilizados, en el que se tome en cuenta el constructo que quieren medir, la confiabilidad y validez de los instrumentos en las poblaciones a estudiar. Un estudio reporta una revisión en la que sintetiza la literatura referente a temperamento e incluye: el concepto de temperamento según diferentes autores, la etapa del niño e instrumento de medición recomendado. Al tomar en cuenta criterios específicos, podría favorecer a presentar resultados auténticos y estandarizados de la población preescolar ${ }^{(19)}$.

La literatura revisada refiere que el componente del temperamento autorregulación muestra una asociación con el incremento de IMC en los niños preescolares, específicamente las habilidades de autorregulación: regulación emocional y la demora a la gratificación. Esta situación puede darse debido a que recientes investigaciones que se encuentran alrededor del estudio de la conducta alimentaria refieren que los procesos complejos de regulación de la energía y la regulación de recompensa, podrían revelar esas diferencias individuales en la regulación energética, preferencias 
en el sabor y el comportamiento de búsqueda de recompensa relacionado con la comida ${ }^{(18)}$. Dentro de las limitaciones en esta revisión se encuentran que no se pudieron realizar comparaciones entre los resultados y cierre de conclusiones de los mismos, pues cada uno de los autores decidieron utilizar diferentes instrumentos o procedimientos para medir el temperamento, además que fueron pocos los artículos que incluían a población hispana, por lo que, podrían haber sido omitidos datos correspondientes a esta población.

\section{CONCLUSIÓN}

La literatura existente es aún insuficiente para establecer la relación entre el temperamento y el incremento del IMC en el niño preescolar, lo que imposibilita, ser concluyente, ya que aún algunos de los estudios muestran información contradictoria, se recomienda que se continúe realizando investigación, pero que se establezcan criterios semejantes para la medición del temperamento, en los que se incluyan la diferencias entre la reactividad atencional y la autorregulación. Además se sugiere continuar con el estudio del temperamento con variables parentales como los estilos de alimentación y características psicológicas como la sensibilidad, pues parece ser que al interactuar en conjunto incrementan el poder predictivo del IMC en el preescolar. Finalmente, solo se puede concluir que este estudio es una oportunidad de ampliar y difundir el conocimiento sobre el temperamento y el incremento del IMC en el niño preescolar, mismo que pareciera tener un impacto significativo y prometedor en la explicación de conductas de alimentación precursoras de la obesidad infantil.

\section{REFERENCIAS}

1. Organización Mundial de la Salud. [OMS].Sobrepeso y Obesidad. 2015. [acceso en 11 de septiembre 2015]. Disponible en: http:// www.who.int/mediacentre/factsheets/fs311/es/

2. Tovar A, Hennessy E, Pirie A, Must A, Gute DM, Hyatt RR, et al. Feeding styles and child weight status among recent immigrant mother-child dyads. International Journal of Behavioral Nutrition \& Physical Activity. 2012; 9: 62-81. Disponible en: http://www.ncbi.nlm.nih.gov/pmc/articles/PMC3439673/

3. Khandpur N, Blaine R, Orlet J, Davison K. Fathers' child feeding practices: A review of the evidence. Appetite. 2014; 78:110-121

4. Anzman-Frasca S, Stifter C, Birch LL. Temperament and childhood obesity risk: A review of the literature. Journal of Developmental \& Behavioral Pediatrics. 2012; 33:732-745.

5. Wu T, Dixon WE, Dalton W, Tudivier F, Liu X. Join Effects of child temperament and maternal sensitivity on the development of childhood obesity. Matern child health J. 2011; 15: 469-477.

6. Frankel LA, Hughes S, O'Connor T, Power T, Fisher J, Hazen N. Parental Influences on Children'a Self-regulation of energy intake: insights from developmental literature on emotion regulation. Journal of Obesity. 2012:1-12.

7. Rothbart M. Temperament, Development, and Personality. SAGE journal. 2007; 16 (4): 207-212.

8. Rothbart MK, Derryberry D. Development of individual differences in temperament. In: Lamb M, Brown A, editors. Advances in developmental psychology. NJ Erlbaum: Hillsdale; 1981. p. 37-86. 
9. Graziano PA, Kelleher R, Calkins SD, Keane SP, Brien MO. Predicting weight outcomes in preadolescence: the role of toddlers' self-regulation skills and the temperament dimension of pleasure. Int J Obes (Lond). 2013; 37(7): 937-942. Disponible en: http://www.ncbi.nlm.nih.gov/pmc/articles/PMC3543516/

10. Faith MS, Hittner JB. Infant temperament and eating style predict change in standardized weight status and obesity risk at 6 years of age. Int $\mathrm{J}$ Obesity. 2010; 34:1515-1523.

11. Graziano PA, Calkins SD, Keane SP. Toddler self-regulation skills predict risk for pediatric obesity. Int $J$ Obes. 2010;34:633-41. Disponible en: http://www.ncbi.nIm.nih.gov/pmc/articles/PMC2854309/

12. Bergmeier $H$, Skouteris $H$, Horwood $S$, Hooley $M$, Richardson B. Child temperament and maternal predictors of preschool children's eating and body mass index. Aprospective study. Appetite. 2014; 14: 125-132.

13. Haycraft E, Farrow C, Meyer C, Powell F, Blissett J. Relationships between temperament and eating behaviours in young children. Appetite; 53 (3): 689692.

14. Moher D, Liberati A, Tetzlaff J, Altman DG. The PRISMA Group Preferred Reporting Items for Systematic Reviews and Meta-Analyses: The PRISMA Statement. PLoS Med. 2009; 6(7): e1-97.doi:10.1371/journal.pmed1000097. Disponible en: http://www.prismastatement. org/PRISMAS tatement/PRISMAS tatement.aspx

15. Hughes SO, Shewchuk RM, Baskin ML, Nicklas TA, Qu H. Indulgent feeding style and children's weight status in preschool. Journal of Developmental and Behavioral Pediatrics. 2008; 29:403-410. Disponible en: http://www. ncbi. nlm.nih.gov/pmc/articles/PMC2769986/

16. Tan CC, Holub SC. Children's self-regulation in eating: associations with inhibitory control and parents's feeding behavior. J Pediatr Psychol. 2011; 36(3): 340-345.

17. Francis LA, Susman EJ. Self-regulation and rapid weight gain in children from age 3 to 12 years. Arch Pediatr Adolesc Med. 2009; 163(4): 297-302.

18. Gahagan S. The development of eating behavior-biology context. J Dev Behav Pediatr. 2012; 33(3): 261-271. Disponible en: http://www.ncbi.nlm.nih. Gov/pmc/articles/PMC3426439/

19.Zentner M, Bates J. Child temperament: a integrative review of concepts, research programs, and measures. European Journal of Developmental Science. 2008; 2 (1/2): 7-37. Disponible en: http://www.unige.ch/fapse/emotion/tests/temperament/publications/ejds 0201 ze ntner.pdf

Recibido: 27 de junio 2016;

Aceptado: 3 de septiembre 2016

ISSN 1695-6141

(C) COPYRIGHT Servicio de Publicaciones - Universidad de Murcia 